\title{
Large-scale energy spectra in surface quasi-geostrophic turbulence
}

\author{
By CHUONG V. TRAN $†$ AND JOHN C. BOWMAN \\ Department of Mathematical and Statistical Sciences, \\ University of Alberta, Edmonton, Alberta, Canada, T6G 2G1
}

(Received 12 May 2004 and in revised form 05 November 2004)

The large-scale energy spectrum in two-dimensional turbulence governed by the surface quasi-geostrophic (SQG) equation

$$
\partial_{t}(-\Delta)^{1 / 2} \psi+J\left(\psi,(-\Delta)^{1 / 2} \psi\right)=\mu \Delta \psi+f
$$

is studied. The nonlinear transfer of this system conserves the two quadratic quantities $\Psi_{1}=\left\langle\left[(-\Delta)^{1 / 4} \psi\right]^{2}\right\rangle / 2$ and $\Psi_{2}=\left\langle\left[(-\Delta)^{1 / 2} \psi\right]^{2}\right\rangle / 2$ (kinetic energy), where $\langle\cdot\rangle$ denotes a spatial average. The energy density $\Psi_{2}$ is bounded and its spectrum $\Psi_{2}(k)$ is shallower than $k^{-1}$ in the inverse-transfer range. For bounded turbulence, $\Psi_{2}(k)$ in the low-wavenumber region can be bounded by $C k$ where $C$ is a constant independent of $k$ but dependent on the domain size. Results from numerical simulations confirming the theoretical predictions are presented.

\section{Introduction}

The dynamics of a three-dimensional stratified rapidly rotating fluid is characterized by the geostrophic balance between the Coriolis force and pressure gradient. The nonlinear dynamics governed by the first-order departure from this linear balance is known as quasigeostrophic dynamics and is inherently three-dimensional. The theory of quasi-geostrophy is interesting and the research performed on this subject constitutes a rich literature (see, for example, Charney 1948, 1971; Rhines 1979; Pedlosky 1987). This theory renders a variety of two-dimensional models that are appealing for their relative simplicity and yet sufficiently sophisticated to capture the underlying dynamics of geophysical fluids. One such model, the so-called surface quasi-geostrophic (SQG) equation, is the subject of the present study.

Quasi-geostrophic flows can be described in terms of the geostrophic streamfunction $\psi(\boldsymbol{x}, t)$. The vertical dimension $z$ is usually taken to be semi-infinite and the horizontal extent may be either bounded or unbounded. Normally, decay conditions are imposed as $z \rightarrow \infty$. At the flat surface boundary $z=0$, the vertical gradient of $\psi(\boldsymbol{x}, t)$ matches the temperature field $T(\boldsymbol{x}, t)$, i.e. $\left.T(\boldsymbol{x}, t)\right|_{z=0}=\left.\partial_{z} \psi(\boldsymbol{x}, t)\right|_{z=0}$. For flows with zero potential vorticity, this surface temperature field can be identified with $(-\Delta)^{1 / 2} \psi$, where $\Delta$ is the (horizontal) two-dimensional Laplacian. Here, the operator $(-\Delta)^{1 / 2}$ is defined by $(-\Delta)^{1 / 2} \widehat{\psi}(\boldsymbol{k})=k \widehat{\psi}(\boldsymbol{k})$, where $k=|\boldsymbol{k}|$ is the wavenumber and $\widehat{\psi}(\boldsymbol{k})$ is the Fourier transform of $\psi(\boldsymbol{x})$. The conservation equation governing the advection of the temperature $(-\Delta)^{1 / 2} \psi$ by the surface flow is (Blumen 1978; Pedlosky 1987; Pierrehumbert, Held

\footnotetext{
$\dagger$ Present address: Mathematics Institute, University of Warwick, Coventry CV4 7AL, UK
} 
\& Swanson 1994; Held et al. 1995)

$$
\partial_{t}(-\Delta)^{1 / 2} \psi+J\left(\psi,(-\Delta)^{1 / 2} \psi\right)=0
$$

where $J(\varphi, \phi)=\partial_{x} \varphi \partial_{y} \phi-\partial_{y} \varphi \partial_{x} \phi$. This equation is known as the SQG equation.

In this paper a forced-dissipative version of (1.1) is studied. A dissipative term of the form $\mu \Delta \psi$, where $\mu>0$, which results from Ekman pumping at the surface, is considered (Constantin 2002; Tran 2004). Since $(-\Delta)^{1 / 2} \psi$ is the advected quantity, this physical dissipation mechanism corresponds to the (hypoviscous) dissipation operator $\mu(-\Delta)^{1 / 2}$. The dissipation coefficient $\mu$ has the dimension of velocity and is not vanishingly small in the atmospheric context (Constantin 2002). The system is assumed to be driven by a forcing $f$, for which the spectral support is confined to wavenumbers $k \geq s>0$ (in bounded turbulence, wavenumber zero is replaced by the minimum wavenumber). Thus, the forced-dissipative SQG equation can be written as

$$
\partial_{t}(-\Delta)^{1 / 2} \psi+J\left(\psi,(-\Delta)^{1 / 2} \psi\right)=\mu \Delta \psi+f .
$$

It is customary in the classical theory of turbulence to consider a doubly periodic domain of size $L$; the unbounded case is obtained via the limit $L \rightarrow \infty$.

The Jacobian operator $J(\cdot, \cdot)$ admits the identities

$$
\langle\chi J(\varphi, \phi)\rangle=-\langle\varphi J(\chi, \phi)\rangle=-\langle\phi J(\varphi, \chi)\rangle,
$$

where $\langle\cdot\rangle$ denotes the spatial average. As a consequence, the nonlinear term in (1.2) obeys the conservation laws

$$
\left\langle\psi J\left(\psi,(-\Delta)^{1 / 2} \psi\right)\right\rangle=\left\langle(-\Delta)^{1 / 2} \psi J\left(\psi,(-\Delta)^{1 / 2} \psi\right)\right\rangle=0
$$

It follows that the two quadratic quantities $\Psi_{\theta}=\left\langle\left|(-\Delta)^{\theta / 4} \psi\right|^{2}\right\rangle / 2=\int \Psi_{\theta}(k) \mathrm{d} k$, where $\theta=1,2$, are conserved by nonlinear transfer. Here, $\Psi_{\theta}(k)$ is defined by $\Psi_{\theta}(k)=k^{\theta} \Psi(k)$, $\Psi(k)$ is the power density of $\psi$ associated with wavenumber $k$ and $\theta$ is a real number. Note that $\Psi_{2}(k)$ is the kinetic energy spectrum and $\Psi_{2}$ is the kinetic energy density.

The simultaneous conservation of two quadratic quantities by advective nonlinearities is a common feature in incompressible fluid systems in two dimensions. Some familiar systems in this category are the Charney-Hasegawa-Mima equation (Hasegawa \& Mima 1978; Hasegawa, Maclennan \& Kodama 1979) and the class of $\alpha$ turbulence equations (Pierrehumbert et al. 1994), which includes both the Navier-Stokes and the SQG equations. These conservation laws, together with the scale-selectivity of the dissipation and unboundedness of the domain, are the building block of the classical dual-cascade theory (Fjørtoft 1953; Kraichnan 1967, 1971; Leith 1968; Batchelor 1969). This theory, when applied to the present case, implies that $\Psi_{1}$ cascades to low wavenumbers (inverse cascade) and $\Psi_{2}$ cascades to high wavenumbers (direct cascade). For some recent discussion on the possibility of a dual cascade in various two-dimensional systems, including the NavierStokes and SQG equations, see Tran \& Shepherd (2002), Tran \& Bowman (2003b,2004) and Tran (2004) The inverse cascade toward wavenumber $k=0$ would eventually evade viscous dissipation altogether because the spectral dissipation rate vanishes as $k \rightarrow 0$. Hence, according to the classical picture, $\Psi_{1}$ necessarily grows unbounded, by a steady growth rate $\mathrm{d} \Psi_{1} / \mathrm{d} t>0$, as $t \rightarrow \infty$. Strictly speaking, one may have to address the possibility of a dissipated inverse cascade, i.e. one for which the dissipation of $\Psi_{1}$ occurs at scales much larger than the forcing scale and for which $\mathrm{d} \Psi_{1} / \mathrm{d} t$ has a zero time mean. Such a cascade is not a plausible scenario (and is not the traditional undissipated inverse cascade) in fluid systems, dissipated by a single viscous operator, where the viscous dissipation rate diminishes toward the large scales. A discussion of this issue can be found in Tran (2004), 
In this study, upper bounds are derived for the time averages of the kinetic energy density $\Psi_{2}$ and of the large-scale spectrum $\Psi_{2}(k)$. These bounds are derived from the governing equation, involving simple but rigorous estimates. The bound on $\Psi_{2}$ is valid in both unbounded and bounded cases, and a straightforward consequence of this bound is a bound on the energy spectrum, which also applies to both unbounded and bounded turbulence. Another bound on the large-scale energy spectrum is derived by estimating the nonlinear triple-product term representing the inverse transfer of $\Psi_{1}$. This result applies to bounded turbulence since upper bounds for the triple-product term are inherently domain-size dependent. The difficulties of extending this result to the unbounded case are discussed. Some numerical results confirming the theoretical predictions are presented.

\section{Bounded dynamical quantities}

A notable feature of unbounded incompressible fluid turbulence in two dimensions is the appearance of infinite quadratic quantities (per unit area): namely, the kinetic energy density $\Psi_{2}$ for Navier-Stokes turbulence and $\Psi_{1}$ for the SQG case. According to the classical theory (applied to the SQG case), a (steady) injection of $\Psi_{1}$, applied around some finite wavenumber $s$, cascades to ever-larger scales, leading to an unbounded growth of $\Psi_{1}$ (this is presumably the case for the general quadratic invariant $\Psi_{\alpha}$ in the so-called $\alpha$ turbulence; $c f$. Tran 2004). In other words, if the classical inverse cascade is realizable, unbounded incompressible fluid turbulence in two dimensions constitutes an ill-posed problem, in the sense that a key quadratic invariant becomes infinite. Of course, there still exist finite quadratic quantities, in particular the dissipation agent for each quadratic invariant. This section is concerned with these quantities.

On multiplying (1.2) by $\psi$ and $(-\Delta)^{1 / 2} \psi$ and taking the spatial average of the resulting equations, noting from the conservation laws that the nonlinear terms identically vanish, one obtains evolution equations for $\Psi_{1}$ and $\Psi_{2}$,

$$
\begin{aligned}
& \frac{\mathrm{d}}{\mathrm{d} t} \Psi_{1}=-2 \mu \Psi_{2}+\langle f \psi\rangle, \\
& \frac{\mathrm{d}}{\mathrm{d} t} \Psi_{2}=-2 \mu \Psi_{3}+\left\langle f(-\Delta)^{1 / 2} \psi\right\rangle .
\end{aligned}
$$

Using the Cauchy-Schwarz and geometric-arithmetic mean inequalities, one obtains upper bounds on the injection terms in (2.1) and (2.2):

$$
\begin{aligned}
\langle f \psi\rangle & \leq\left\langle\left|(-\Delta)^{1 / 2} \psi\right|^{2}\right\rangle^{1 / 2}\left\langle\left|(-\Delta)^{-1 / 2} f\right|^{2}\right\rangle^{1 / 2} \leq \mu \Psi_{2}+\mu^{-1} F_{-2}, \\
\left\langle f(-\Delta)^{1 / 2} \psi\right\rangle & \leq\left\langle\left|(-\Delta)^{3 / 4} \psi\right|^{2}\right\rangle^{1 / 2}\left\langle\left|(-\Delta)^{-1 / 4} f\right|^{2}\right\rangle^{1 / 2} \leq \mu \Psi_{3}+\mu^{-1} F_{-1},
\end{aligned}
$$

where the 'integration by parts' rule $\left\langle(-\Delta)^{\theta} \phi \chi\right\rangle=\left\langle(-\Delta)^{\theta^{\prime}} \phi(-\Delta)^{\theta^{\prime \prime}} \chi\right\rangle$, for $\theta=\theta^{\prime}+\theta^{\prime \prime}$, has been used and $F_{\theta}=\left\langle\left|(-\Delta)^{\theta / 4} f\right|^{2}\right\rangle / 2$. Substituting (2.3) in (2.1) and (2.2) yields

$$
\begin{aligned}
\frac{\mathrm{d}}{\mathrm{d} t} \Psi_{1} & \leq-\mu \Psi_{2}+\mu^{-1} F_{-2}, \\
\frac{\mathrm{d}}{\mathrm{d} t} \Psi_{2} & \leq-\mu \Psi_{3}+\mu^{-1} F_{-1} .
\end{aligned}
$$

To avoid unnecessary complications, zero initial conditions are assumed, so that for $T>0$ the time means $\left\langle\mathrm{d} \Psi_{1} / \mathrm{d} t\right\rangle_{t}=\Psi_{1}(T) / T$ and $\left\langle\mathrm{d} \Psi_{2} / \mathrm{d} t\right\rangle_{t}$ are non-negative. One can then deduce upper bounds on the time means $\left\langle\Psi_{2}\right\rangle_{t}$ and $\left\langle\Psi_{3}\right\rangle_{t}$, which are valid regardless of whether or not $\Psi_{1}$ remains finite in the limit $t \rightarrow \infty$ :

$$
\begin{aligned}
& \left\langle\Psi_{2}\right\rangle_{t} \leq \mu^{-2}\left\langle F_{-2}\right\rangle_{t}, \\
& \left\langle\Psi_{3}\right\rangle_{t} \leq \mu^{-2}\left\langle F_{-1}\right\rangle_{t} .
\end{aligned}
$$


For $\theta \in(2,3),\left\langle\Psi_{\theta}\right\rangle_{t}$ is also bounded. Indeed, from the Hölder inequalities $\Psi_{\theta} \leq$ $\Psi_{2}^{3-\theta} \Psi_{3}^{\theta-2}$ (cf. Tran 2004) and $\left\langle\Psi_{2}^{3-\theta} \Psi_{3}^{\theta-2}\right\rangle_{t} \leq\left\langle\Psi_{2}\right\rangle_{t}^{3-\theta}\left\langle\Psi_{3}\right\rangle_{t}^{\theta-2}$, one can deduce from (2.6) and (2.7) that

$$
\left\langle\Psi_{\theta}\right\rangle_{t} \leq\left\langle\Psi_{2}\right\rangle_{t}^{3-\theta}\left\langle\Psi_{3}\right\rangle_{t}^{\theta-2} \leq \mu^{-2}\left\langle F_{-2}\right\rangle_{t}^{3-\theta}\left\langle F_{-1}\right\rangle_{t}^{\theta-2} .
$$

This result implies that for $\theta \in(2,3),\left\langle\Psi_{\theta}\right\rangle_{t}$ is bounded, provided that both $\left\langle F_{-1}\right\rangle_{t}$ and $\left\langle F_{-2}\right\rangle_{t}$ are bounded. This condition is assured if $s>0$ and $F_{0}$ is bounded, a condition normally required of the forcing, because $F_{-2} \leq F_{-1} / s \leq F_{0} / s^{2}$. One may even consider a class of forcing for which $F_{0}=\infty$ and $F_{-2} \leq F_{-1} / s<\infty$.

Upper bounds of the above type on dynamical quantities are rather trivial for bounded turbulence. However, they are important in the unbounded case, for two reasons. First, the scale-selective viscous dissipation allows for the possibility of unbounded growth of certain quadratic quantities toward the low wavenumbers. Hence, rigorous bounds on dynamical quantities are not as abundant as in the bounded case. Second, analytic studies of the nonlinear triple-product transfer function are difficult in unbounded domains. In the absence of pointwise estimates for the spectrum, these bounds are particularly useful for qualitative estimates of the large-scale distribution of energy. For example, Tran (2004) uses inequality (2.6) to argue that the energy spectrum $\Psi_{2}(k)$ should be shallower than $k^{-1}$, as $k \rightarrow 0$.

\section{Large-scale energy spectrum}

In this section, it is shown that the physical laws of SQG dynamics admit only largescale energy spectra shallower than $k^{-1}$. This result is due in part to the fact that the simultaneous conservation of $\Psi_{1}$ and $\Psi_{2}$ allows virtually no kinetic energy to get transferred toward the low wavenumbers, so that only large-scale kinetic energy spectra shallower than $k^{-1}$ are possible.

\subsection{Shell-averaged energy spectrum}

For a given wavenumber $r$, let us denote by $S=S(r)$ the wavenumber shell between $k=r / 2$ and $k=3 r / 2$, i.e. $S(r)=\{\boldsymbol{k}: r / 2 \leq k \leq 3 r / 2\}$. The shell-averaged energy spectrum $\bar{\Psi}_{2}(r)$ over $S(r)$ is defined by

$$
\bar{\Psi}_{2}(r)=\frac{1}{r} \int_{r / 2}^{3 r / 2} \Psi_{2}(k) \mathrm{d} k .
$$

In the present case of a doubly periodic domain of size $L$, the Fourier representation of the stream function is $\psi(\boldsymbol{x})=\sum_{\boldsymbol{k}} \exp \{i \boldsymbol{k} \cdot \boldsymbol{x}\} \widehat{\psi}(\boldsymbol{k})$, where $\boldsymbol{k}=2 \pi L^{-1}\left(k_{x}, k_{y}\right)$ with $k_{x}$ and $k_{y}$ being integers not simultaneously zero. Let $\psi(S)$ denote the component of $\psi$ spectrally supported by $S$, i.e. $\psi(S)=\sum_{\boldsymbol{k} \in S} \exp \{i \boldsymbol{k} \cdot \boldsymbol{x}\} \widehat{\psi}(\boldsymbol{k})$. One has

$$
\sup _{\boldsymbol{x}}|\nabla \psi(S)| \leq \sum_{\boldsymbol{k} \in S} k|\widehat{\psi}(\boldsymbol{k})| \leq\left(\sum_{\boldsymbol{k} \in S} 1 \sum_{\boldsymbol{k} \in S} k^{2}|\widehat{\psi}(\boldsymbol{k})|^{2}\right)^{1 / 2} \leq c L r \Psi_{2}^{1 / 2}(S),
$$

where the Cauchy-Schwarz inequality is used, the sum $\sum_{\boldsymbol{k} \in S} 1=(c L r)^{2}$ is the number of wavevectors in $S, c$ is an absolute constant of order unity and $\Psi_{2}(S)$ is the contribution to the kinetic energy from $S$. 


\subsection{Upper bounds for the energy spectrum}

A simple upper bound for $\bar{\Psi}_{2}(k)$, which is applicable to both the unbounded and bounded cases, can be derived from (2.6). In fact, it follows from (2.6) and (3.1) that

$$
\left\langle\bar{\Psi}_{2}(k)\right\rangle_{t}=\frac{1}{k} \int_{k / 2}^{3 k / 2}\left\langle\Psi_{2}(\kappa)\right\rangle_{t} \mathrm{~d} \kappa \leq \mu^{-2}\left\langle F_{-2}\right\rangle_{t} k^{-1} .
$$

This bound is supposed to apply to $k$ in the inverse-transfer region. For $k$ in the directtransfer region, (2.7) yields

$$
\left\langle\bar{\Psi}_{2}(k)\right\rangle_{t}=\frac{1}{k} \int_{k / 2}^{3 k / 2}\left\langle\Psi_{2}(\kappa)\right\rangle_{t} \mathrm{~d} \kappa \leq \frac{2}{k^{2}} \int_{k / 2}^{3 k / 2}\left\langle\Psi_{3}(\kappa)\right\rangle_{t} \mathrm{~d} \kappa \leq 2 \mu^{-2}\left\langle F_{-1}\right\rangle_{t} k^{-2} .
$$

The upper bound (3.3) suggests that dimensional analysis arguments, which predict a large-scale $k^{-1}$ energy spectrum, are not well justified. If a persistent inverse cascade of $\Psi_{1}$ exists $\left(\mathrm{d} \Psi_{1} / \mathrm{d} t>0\right)$, then the energy $\Psi_{2}$ ought to acquire a value such that $\Psi_{2}<\mu^{-2} F_{-2}$. In the unbounded case, the large-scale energy spectrum then needs to be strictly shallower than $k^{-1}$, to ensure that the dissipation of $\Psi_{1}$ does not increase without bound as the inverse cascade proceeds toward $k=0$. On the other hand, if no inverse cascade of $\Psi_{1}$ exists, then a $k^{-1}$ energy spectrum with limited extent is possible. If viscous dissipation mechanisms with degrees higher than that of the natural dissipation are considered, then the upper bounds derived above are not valid. Nevertheless, diminishing energy transfer towards the lowest wavenumbers appears to be consistent only with spectra shallower than $k^{-1}$ (for low-wavenumber convergence of the energy integral). The numerical results reported in $\S 4$ are well suited to this expectation.

An upper bound for the large-scale energy spectrum, based on the nonlinear transfer term, can be derived for the bounded case. This analysis employs elementary but rigorous estimates of the triple-product term. For $3 k / 2<s$, the evolution of $\Psi_{1}(S(k))$ is governed by

$$
\begin{aligned}
\frac{\mathrm{d}}{\mathrm{d} t} \Psi_{1}(S) & =-\left\langle\psi(S) J\left(\psi,(-\Delta)^{1 / 2} \psi\right)\right\rangle-2 \mu \Psi_{2}(S) \\
& =\left\langle(-\Delta)^{1 / 2} \psi J(\psi, \psi(S))\right\rangle-2 \mu \Psi_{2}(S) \\
& \leq\left\langle\left|(-\Delta)^{1 / 2} \psi \| \nabla \psi\right||\nabla \psi(S)|\right\rangle-2 \mu \Psi_{2}(S) \\
& \leq \sup _{\boldsymbol{x}}|\nabla \psi(S)|\left\langle\left|(-\Delta)^{1 / 2} \psi \|\right| \nabla \psi \mid\right\rangle-2 \mu \Psi_{2}(S) \\
& \leq 2 c L k \Psi_{2}^{1 / 2}(S) \Psi_{2}-2 \mu \Psi_{2}(S) \\
& \leq c^{2} \mu^{-1} L^{2} k^{2} \Psi_{2}^{2}-\mu \Psi_{2}(S) \\
& =c^{2} \mu^{-1} L^{2} k^{2} \Psi_{2}^{2}-\mu k \bar{\Psi}_{2}(k)
\end{aligned}
$$

where the second equality is a consequence of 1.3 and the second last and last inequalities follow from (3.2) and the geometric-arithmetic mean inequality, respectively. It follows that

$$
\left\langle\bar{\Psi}_{2}(k)\right\rangle_{t} \leq c^{2} \mu^{-2} L^{2} k\left\langle\Psi_{2}^{2}\right\rangle_{t} .
$$

A notable feature of (3.6) is its dependence on the fluid domain size. The presence of $L$ in this upper bound is natural: the upper bound $\sup _{\boldsymbol{x}}|\nabla \psi(S)|$, which is associated with the fluid velocity at scales $\approx k^{-1}$, is inherently domain-size dependent. There are no known analytic estimates that allow one to derive an upper bound on the nonlinear transfer function $\left\langle\psi(S) J\left(\psi,(-\Delta)^{1 / 2} \psi\right)\right\rangle$ in terms of 'intensive quantities' only. This difficulty arises not only in the present estimate but also in other analytic estimates of the transfer 
function. In other words, the nonlinear triple-product term is intrinsically domain-size dependent. This problem considerably limits our ability to assess the nonlinear transfer in unbounded systems. Finally, it is worth mentioning that although the upper bound (3.6) has a linear dependence on $k$, it may be more excessive than the bound $\mu^{-2}\left\langle F_{-2}\right\rangle_{t} k^{-1}$ derived earlier (even for very low wavenumbers). The reason is that $L^{2} k \geq k^{-1}$ and the prefactor $c^{2}\left\langle\Psi_{2}^{2}\right\rangle_{t}$ may not be as optimal as $\left\langle F_{-2}\right\rangle_{t}$.

\section{Numerical results}

This section reports results from numerical simulations that illustrate the realization of large-scale spectra shallower than $k^{-1}$. Equation (1.2) is simulated in a doubly periodic square of side $2 \pi$, where the forcing $\widehat{f}(\boldsymbol{k})$ is nonzero only for those wavevectors $\boldsymbol{k}$ having magnitudes lying in the interval $K=[59,61]$ :

$$
\widehat{f}(\boldsymbol{k})=\frac{\epsilon}{N} \frac{\widehat{\psi}(\boldsymbol{k})}{2 \Psi_{1}(k)} .
$$

Here $\epsilon=1$ is the constant energy injection rate and $N$ is the number of distinct wavenumbers in $K$. The (constant) injection rate of $\Psi_{1}$ is $\epsilon / s \approx 1 / 60$, where $1 / s \approx 1 / 60$ is the mean of $k^{-1}$ over $K$. This type of forcing was used by Shepherd (1987), Tran (2004) and Tran \& Bowman (2004) in numerical simulations of a large-scale zonal jet on the so-called beta-plane and of Navier-Stokes turbulence. The attractive aspect of (4.1), as noted in Shepherd (1987) is that it is steady. Dealiased $683^{2}$ and $1365^{2}$ pseudospectral simulations (1024 $4^{2}$ and $2048^{2}$ total modes) were performed. Three dissipative forms were considered: $2.5 \times 10^{-2} \Delta \psi,-4 \times 10^{-4}(-\Delta)^{3 / 2} \psi$, and $-6 \times 10^{-6} \Delta^{2} \psi+\mu \Delta \psi$ for several values of $\mu$. The first case represents the natural dissipation of the SQG dynamics due to Ekman pumping, as mentioned earlier. The second case represents thermal (molecular) diffusion since $(-\Delta)^{1 / 2} \psi$ is equivalent to the fluid temperature. The third case - the mixed hyperviscous/Ekman dissipation form - is considered in order to demonstrate that even slight amounts of Ekman damping will inhibit the formation of an inverse cascade. Unlike Smith et al. (2002), the case of mechanical friction $\left[\propto(-\Delta)^{1 / 2} \psi\right]$ was not considered. The higher resolution was used for the first (natural dissipation) case and the lower resolution was used for the second and third cases. All simulations were initialized with the spectrum $\Psi_{2}(k)=10^{-5} \pi k /\left(60^{2}+k^{2}\right)$.

Figure 1] shows the time-averaged steady-state kinetic energy spectrum for the case of the natural dissipation term $2.5 \times 10^{-2} \Delta \psi$. The dissipation agents of $\Psi_{1}$ and $\Psi_{2}$ are, respectively, $\Psi_{2}$ (energy) and $\Psi_{3}$. The value of the energy, 0.3333 , implies that the dissipation of $\Psi_{1}$, averaged in the same period, is 0.01666 . This amounts to virtually all of the injection rate $1 / 60$. Hence, there exists no inverse cascade of $\Psi_{1}$ to the large scales and both $\Psi_{1}$ and $\Psi_{2}$ are steady. The small-scale energy spectrum scales as $k^{-3.5}$, so that the spectrum $\Psi_{3}(k)$ scales as $k^{-2.5}$. This scaling means that the energy dissipation occurs mainly around the forcing region and is consistent with the bound (3.4).

Unlike Navier-Stokes turbulence, for which the inverse energy cascade is robust and can be simulated at relatively low resolution, it was noticed that no choice for the value of $\mu$ at the present resolution could be used to simulate an inverse cascade of $\Psi_{1}$. It is not known whether an inverse cascade of $\Psi_{1}$ is realizable at higher resolutions, using a smaller value of $\mu$. Nevertheless, this observation suggests that $\Psi_{1}$ is 'reluctant' to cascade to the large scales, as compared with the more robust inverse energy cascade in Navier-Stokes turbulence.

Figure 2 shows the kinetic energy spectrum averaged between $t=37.3$ and $t=38.7$, 


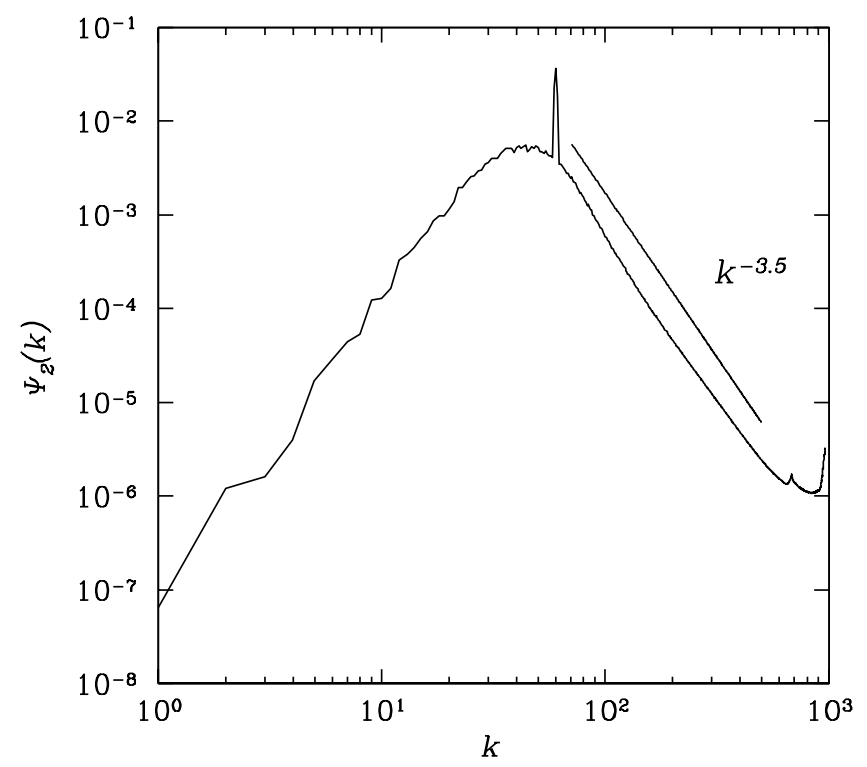

Figure 1 . The time-averaged steady-state energy spectrum $\Psi_{2}(k)$ vs. $k$ for the dissipation term $2.5 \times 10^{-2} \Delta \psi$

for a lower viscous degree. The dissipation agents of $\Psi_{1}$ and $\Psi_{2}$ are, respectively, $\Psi_{3}$ and $\Psi_{4}$ (enstrophy). The value of $\Psi_{3}$ is 20 , implying that the dissipation of $\Psi_{1}$ is $1.6 \times 10^{-2}$. This amounts to about $96 \%$ of the injection rate $1 / 60$. The inverse cascade then carries only a few percent of the injection of $\Psi_{1}$ to the large scales.

The small-scale energy spectrum scales as $k^{-4.5}$, so that the enstrophy spectrum $\Psi_{4}(k)$ scales as $k^{-2.5}$. Most of the energy dissipation occurs around the forcing region, consistent with a 'weak' inverse cascade (one that does not carry virtually all of the injection of $\Psi_{1}$ toward $k=0 ; c f$. Tran and Bowman 2004, Tran 2004). No direct cascade is possible for bounded turbulence in equilibrium or for unbounded turbulence in the presence of a weak inverse cascade.

Similarly, Figure 3 shows the kinetic energy spectrum averaged between $t=15.7$ and $t=16.5$ for the mixed dissipation $-6 \times 10^{-6} \Delta^{2} \psi+\mu \Delta \psi$, using three different values of $\mu$. When $\mu=0$, the dissipation agents of $\Psi_{1}$ and $\Psi_{2}$ are, respectively, $\Psi_{4}$ (enstrophy) and $\Psi_{5}$. The value of the enstrophy, 1208, implies that the dissipation of $\Psi_{1}$ is $1.45 \times 10^{-2}$, amounting to about $87 \%$ of the injection rate $1 / 60$. The small-scale energy spectrum scales as $k^{-5}$, so that $\Psi_{5}(k)$ scales as $k^{-2}$. Again, this scaling means that most of the energy dissipation occurs around the forcing region and that the inverse cascade is weak. We note that as $\mu$ is increased, the inverse cascade becomes increasingly weak. We emphasize this behaviour by plotting in Fig. 4 the inverse cascade strength $r=1-2 s\left(\mu \Psi_{2}+6 \times 10^{-6} \Psi_{4}\right) / \epsilon$ for six different values of $\mu$.

Unlike Navier-Stokes turbulence, for which the enstrophy acquires its near-equilibrium value once a discernible inverse-transfer range has formed, the energy in SQG turbulence can remain significantly less than its equilibrium value until a very wide inverse-transfer range has developed. For example, for a one-decade Navier-Stokes inverse-transfer range (achievable in numerical simulations), the enstrophy acquires $95 \%$ of its projected equilibrium value (calculated with a $k^{-5 / 3}$ energy spectrum extrapolated to $k=0$ ). On the other hand, for a one-decade SQG inverse-transfer range, the energy acquires only $66 \%$ of its projected equilibrium value (calculated with a $k^{-0.7}$ energy spectrum extrapolated to 


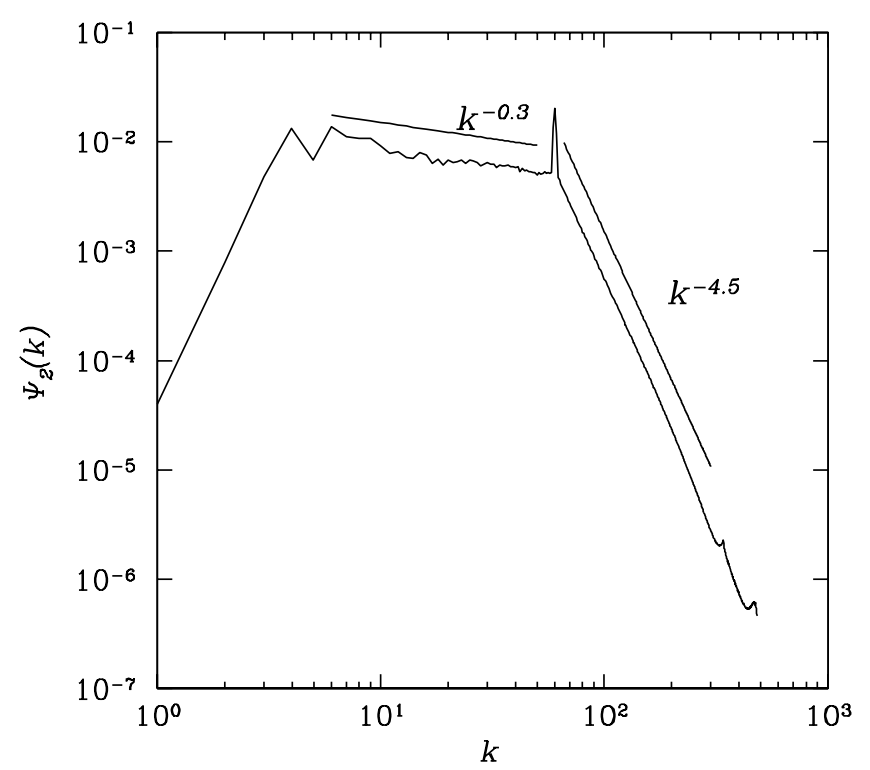

Figure 2. The quasisteady energy spectrum $\Psi_{2}(k)$ vs. $k$ averaged between $t=37.3$ and $t=38.7$ for the dissipation term $-4 \times 10^{-4}(-\Delta)^{3 / 2} \psi$.

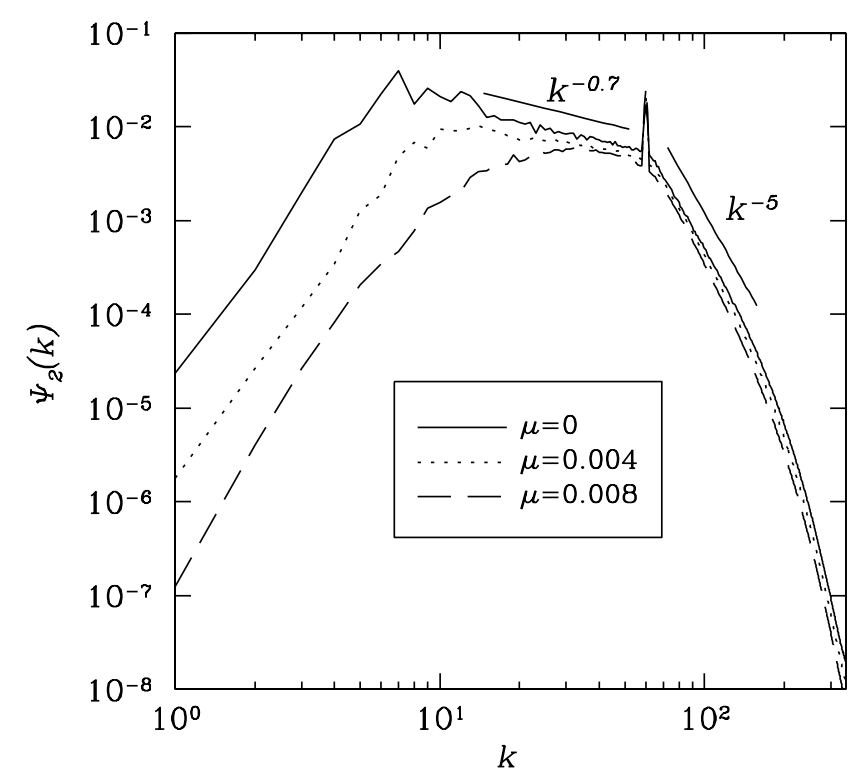

FIgURE 3. The quasisteady energy spectrum $\Psi_{2}(k)$ vs. $k$ averaged between $t=15.67$ and $t=16.52$ for the dissipation term $-6 \times 10^{-6} \Delta^{2} \psi+\mu \Delta \psi$, using three different values of $\mu$.

$k=0$, as realized in the present simulations; $c f$. the $\mu=0$ case of Figure 3). This means that one needs a considerably wider inverse-transfer region for SQG turbulence than for Navier-Stokes turbulence, in order to approach a quasi-steady state. This problem is in addition to the resolution limitations at the small scales for both cases.

Due to the steep spectrum in the inverse-transfer region, the energy in the $\mu=0$ case of Figure 3 has not acquired a value considerably close to its equilibrium value. This means that the system is still well within the transient phase, However, the dissipation 


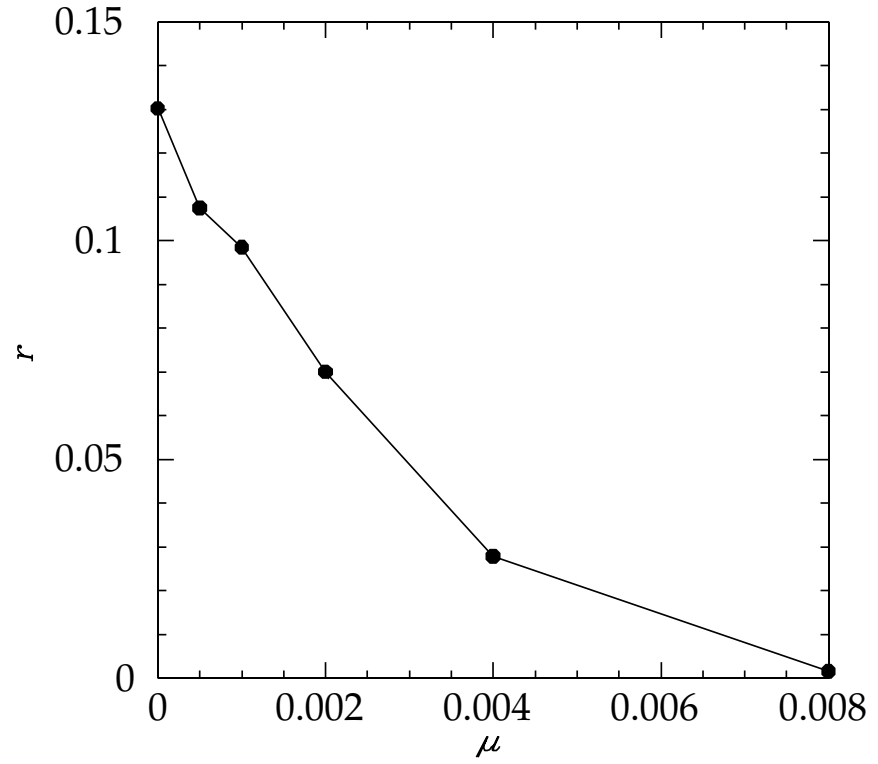

Figure 4. The decay of the inverse cascade strength $r$ for the dissipation term $-6 \times 10^{-6} \Delta^{2} \psi+\mu \Delta \psi$ as $\mu$ is increased.

of $\Psi_{1}$ (proportional to the enstrophy) cannot grow considerably (without significant change to the existing spectrum), because of the high degree of viscosity, which makes the dissipation of $\Psi_{1}$ relatively insensitive to growth of the large-scale energy.

\section{Conclusion and discussion}

In this paper, the kinetic energy density of SQG turbulence and its large-scale spectrum have been studied. For the unbounded case, upper bounds are derived for the time means of the kinetic energy density and of the large-scale energy spectrum, averaged over a narrow window of wavenumbers. Another result is an upper bound on the the time mean of the large-scale energy spectrum, which is derived for the bounded case. Numerical results confirming the predicted slopes of the large-scale energy spectrum are presented and discussed.

An important feature in SQG turbulence that gives rise to the rigorous upper bound on the time mean of the kinetic energy density in the unbounded case is that the kinetic energy is the dissipation agent of the inverse-cascading candidate $\Psi_{1}$. This fact is due to the hypoviscous nature of the dissipation operator $(-\Delta)^{1 / 2}$, a natural physical dissipation mechanism of SQG dynamics (Ohkitani 1997; Constantin 2002; Tran 2004). If $(-\Delta)^{1 / 2}$ is replaced by an operator of the form $(-\Delta)^{\eta}$, where $\eta>1 / 2$, then the simple analysis of Section 2 fails to show that the time mean of the energy density $\left\langle\Psi_{2}\right\rangle_{t}$ is bounded, although it may remain so for low degrees of viscosity $\eta$. The reason is that the amount of energy getting transferred to wavenumbers lower than a given wavenumber $k$ decreases at least as rapidly as $k$, so that the spectral dissipation rate $\propto k^{2 \eta}$, a consequence of the dissipation operator $(-\Delta)^{\eta}$, may be sufficiently strong to balance the diminishing inverse energy transfer and keep the energy from growing unbounded.

Numerical simulations of SQG turbulence were performed, using the natural dissipation operator $(-\Delta)^{1 / 2}$ and two viscous operators $\Delta$ and $(-\Delta)^{3 / 2}$. The results show large-scale energy spectra shallower than $k^{-1}$, consistent with the theoretical prediction. 
There have been attempts to explain, within the context of SQG turbulence (Constantin 2002; Tung \& Orlando 2003), the kinetic energy spectra observed in the laboratory experiment of Baroud (2002) and in the atmosphere. In the former case, the turbulence in a rotating tank is driven at a sufficiently small scale to allow for a wide inverse-transferring range. A $k^{-2}$ spectrum extending over nearly two wavenumber decades lower than the forcing wavenumber is observed. In the latter case, a $k^{-5 / 3}$ spectrum is observed in the mesoscales (see Frisch 1995 and Tung \& Orlando 2003 and references therein), which correspond to wavenumbers higher (lower) than the forcing wavenumber if the energy released from baroclinic instability (thunderstorms) is considered to be the driving force. The -2 power-law scaling observed in Baroud (2002) for the wavenumber range lower than the forcing wavenumber is excessively steeper than the permissible scalings derived in this work. The $-5 / 3$ slope in the atmosphere is either steeper (if considered to be on the wavenumber range lower than the forcing wavenumber) or shallower (if considered to be on the wavenumber range higher than the forcing wavenumber) than the permissible slopes. According to the present analysis, these data cannot be attributed to SQG turbulence.

We would like to thank two anonymous referees for their comments, which were helpful in improving this manuscript. This work was funded by a Pacific Institute for the Mathematical Sciences Postdoctoral Fellowship, an Alexander von Humboldt Research Fellowship, and the Natural Sciences and Engineering Research Council of Canada.

\section{REFERENCES}

Baroud, C.N., Plapp, B.B, She, Z.-S. \& Swinney H.L. 2002 Anomalous self-similarity in a turbulent rapidly rotating fluid. Phys. Rev. Lett. 88, 114501.

BAtChelor, G.K. 1969 Computation of the energy spectrum in homogeneous, two-dimensional turbulence. Phys. Fluids 12, II 233-239.

Blumen, W. 1978 Uniform potential vorticity flow, Part I: Theory of wave interactions and two-dimensional turbulence. J. Atmos. Sci. 35, 774-783.

Charney, J.G. 1948 On the scale of atmospheric motions. Geofys. Publ. 17, 3-17.

Charney, J.G. 1971 Geostrophic turbulence. J. Atmos. Sci. 28, 1087-1095.

Constantin, P. 2002 Energy spectrum of quasigeostrophic turbulence. Phys. Rev. Lett. 89, 184501.

Constantin, P., Majda, A.J. \& Tabak, E.G. 1994 Singular front formation in a model for quasigeostrophic flow. Phys. Fluids 6, 9-11.

Constantin, P., Majda, A.J. \& TABak, E.G. 1994 Formation of strong fronts in the 2-D quasigeostrophic thermal active scalar. Nonlinearity 7, 1495-1533.

FJøRTOFт, R. 1953 On the changes in the spectral distribution of kinetic energy for twodimensional, nondivergent flow. Tellus 5, 225-230.

Frisch, U. 1995 Turbulence: The Legacy of A. N. Kolmogorov. Cambridge University Press, Cambridge.

Hasegawa, A. \& Mima, K. 1978 Pseudo-three-dimensional turbulence in magnetized nonuniform plasma. Phys. Fluids 21, 87-92.

Hasegawa, A., Maclennan, C.G. \& Kodama, Y. 1979 Nonlinear behavior and turbulence spectra of drift waves and Rossby waves. Phys. Fluids 22, 2122-2129.

Held, I.M., Pierrehumbert, R.T., Garner, S.T. \& Swanson, K.L. 1995 Surface quasigeostrophic dynamics. J. Fluid Mech. 282, 1-20.

Kraichnan, R.H. 1967 Inertial ranges in two-dimensional turbulence. Phys. Fluids 10, 14171423.

KRAiChnAN, R.H. 1971 Inertial-range transfer in two- and three-dimensional turbulence. J. Fluid Mech. 47, 525-535.

Leith, C.E. 1968 Diffusion approximation for two-dimensional turbulence. Phys. Fluids 11, 671-673. 
OHKITANi, K. \& YAmADA, M. 1997 Inviscid and inviscid-limit behavior of a surface quasigeostrophic flow. Phys. Fluid 9, 876-882.

Pedlosky, J. 1987 Geophysical Fluid Dynamics. 2nd Edition, Springer, New York, 1987.

Pierrehumbert, R.T., Held, I.M., Swanson, K.L. 1994 Spectra of local and nonlocal twodimensional turbulence. Chaos Solitons Fract. 4, 1111-1116.

Rhines, P.B. 1979 Geostrophic turbulence. Ann. Rev. Fluid Mech. 11, 401-441.

ShepherD, T.G. 1987 Rossby waves and two-dimensional turbulence in a large-scale zonal jet. J. Fluid Mech. 183, 467-509.

Smith, K.S., Boccaletti, G., Henning, C.C., Marinov, I., Tam, C.Y., Held, I.M. \& VAlLIS, G.K. 2002 Turbulent diffusion in the geostrophic inverse cascade. J. Fluid Mech. 469, 13-48.

Tran, C.V. 2004 Nonlinear transfer and spectral distribution of energy in $\alpha$ turbulence. Physica D 191, 137-155.

Tran, C.V. \& Bowman, J.C. 2004 Robustness of the inverse cascade in two-dimensional turbulence. Phys. Rev. E 69, 036303.

Tran, C.V. \& Bowman, J.C. 2003a On the dual cascade in two-dimensional turbulence. Physica D 176, 242-255.

Tran, C.V. \& Bowman, J.C. 2003b Energy budgets in Charney-Hasegawa-Mima and surface quasigeostrophic turbulence. Phys. Rev. E 68, 036304.

Tran, C.V. \& Shepherd, T.G. 2002 Constraints on the spectral distribution of energy and enstrophy dissipation in forced two-dimensional turbulence. Physica D 165, 199-212.

Tung, K.K. \& Orlando, W.W. 2003 On the differences between 2D and QG turbulence. Discrete Contin. Dyn. Syst. Ser. B 3, 145-162. 